\title{
Resonance enhancement of first- and second-order coherent phonons in metallic single-walled carbon nanotubes
}

\author{
K. Sato, ${ }^{1}$ K. Tahara, ${ }^{1}$ Y. Minami, ${ }^{1}$ I. Katayama, ${ }^{1}$ M. Kitajima,${ }^{1,3,4}$ H. Kawai, ${ }^{2}$ K. Yanagi, ${ }^{2}$ and J. Takeda ${ }^{1}$ \\ ${ }^{1}$ Graduate School of Engineering, Yokohama National University, Yokohama 240-8501, Japan \\ ${ }^{2}$ Department of Physics, Tokyo Metropolitan University, Hachioji 192-0397, Japan \\ ${ }^{3}$ LxRay Company, Ltd., Nishinomiya 663-8172, Japan \\ ${ }^{4}$ Department of Applied Physics, National Defense Academy, Yokohama 239-8686, Japan
}

(Received 29 August 2014; revised manuscript received 10 December 2014; published 29 December 2014)

\begin{abstract}
High-frequency coherent phonons resonantly excited in metallic single-walled carbon nanotubes (M-SWCNTs) were investigated via spectrally resolved pump-probe spectroscopy using 7.5-fs laser pulses. In addition to first-order coherent phonons such as radial breathing mode (RBM) and $M$ and $G$ modes, we clearly observed second-order high-frequency coherent phonons of $2 D$ and $2 G$ modes, which can be regarded as squeezed phonons. We found that the amplitudes of the RBM, $G$ and $2 D$ modes were resonantly enhanced at specific wavelengths: the maximum resides at a wavelength whose energy is smaller than that of the van Hove singularities in M-SWCNTs by an amount corresponding to the phonon energy. Furthermore, the $2 D$ mode has stronger enhancement than the other first-order Raman modes. These results indicate that the enhancement originates from a Stokes-stimulated Raman-scattering process at van Hove singularities and that efficient resonance enhancement occurs for the $2 D$ mode, possibly through double resonance due to the trigonal warping effect and strong electron-phonon coupling due to the Kohn anomaly.
\end{abstract}

DOI: 10.1103/PhysRevB.90.235435

PACS number(s): 61.48.De, 42.65.Ky, 42.70.Mp, 78.20.Ci

Coherent phonon spectroscopy is well established as a powerful tool in investigating electron-phonon coupling dynamics in various materials [1-3]. It enables not only the observation of phonon anharmonicity [4] but also the coherent control of phonon dynamics, which has been extensively investigated to date [5-8]. The generation mechanism of coherent phonons has been theoretically addressed: the displacive excitation of coherent phonons mechanism is dominant in the resonant case, whereas the impulsively stimulated Raman-scattering process becomes dominant in the nonresonant case $[9,10]$. By changing the pump-probe wavelength, it is thus possible to reveal the transition from a resonant to nonresonant mechanism [11,12]. In contrast to the generation process, the detection process of coherent phonons has been studied only recently; probewavelength resolved coherent phonon spectroscopy may potentially unveil fruitful physics on electron-phonon coupling dynamics [13-16].

Carbon nanotubes (CNTs) exhibit the characteristic resonant effect in optical transitions because of sharp van Hove singularities due to one-dimensional excitonic resonance [see Fig. 1(a)] [17]. Furthermore, the strong electron-phonon coupling originating from the Kohn anomaly is expected to enhance the second-order coherences relevant to second-order Raman modes, namely, the $2 D$ and $2 G$ modes $[18,19]$. Although many researchers have investigated coherent phonons in CNTs, most of them focused on the dynamics of first-order coherent phonons such as the RBM and $G$ mode [7,14,20,21], due to limitations on reducing pulse duration in order to observe second-order high-frequency phonons. Consequently, neither the electron-phonon coupling dynamics accompanied with such phonons nor the probing-process resonance properties of van Hove singularities in coherent phonons have been uncovered.

In the present study, we demonstrate that spectrally resolved coherent phonon spectroscopy using sub-10-fs laser pulses un- veils the characteristic electronic resonance of CNTs. We used metallic single-walled CNTs (M-SWCNTs) with a diameter of $1.4 \mathrm{~nm}$, because the energy of the lowest van Hove singularity $\left[\sim 700 \mathrm{~nm}\right.$, see $E_{11}^{\mathrm{M}}$ in Fig. 1(a)] falls into the spectral region of the laser $(680-1050 \mathrm{~nm})[17,22]$. This simplifies the discussion of the resonant effect in coherent phonon CNT experiments. Our results indicate that the resonance of the lowest van Hove singularity, via the coherent stimulated Stokes Raman-scattering process, plays an important role in the strong enhancement of first- and second-order high-frequency phonon modes. This enhancement is stronger in the $2 D$ mode, indicating a selective enhancement of higher-order modes with strong electron-phonon coupling.

M-SWCNTs with an average diameter of $1.4 \pm 0.1 \mathrm{~nm}$ were extracted from commercial CNTs (Meijo Nano Carbon Company, Ltd.) using the density-gradient purification method, and were dried to form a film [22-24]. As an example, one of the probable M-SWCNT structures in our sample is shown in Fig. 1(b). The concentration of residual semiconductor nanotubes was estimated to be less than $5 \%$ via absorption spectroscopy. For coherent phonon experiments, we used a Ti:sapphire laser with a 7.5-fs pulse duration (VENTEON PULSE: ONE), with an output power of $200 \mathrm{~mW}$, a spectral bandwidth of $300 \mathrm{~nm}$ ranging from 700 to $1000 \mathrm{~nm}$, and a repetition rate of $90 \mathrm{MHz}$. This broad bandwidth allowed us to examine the resonant properties of coherent phonons via spectrally resolved pump-probe measurements. The output of the laser was separated into two beams; one is used as a pump beam to excite the high-frequency coherent phonons, and the other is used as a probe beam. During the experiments, the pump intensity was kept at $15 \mathrm{~mW}\left(0.05 \mathrm{~mJ} / \mathrm{cm}^{2}\right)$, which is low enough to avoid damaging samples, while the probe intensity was tuned to one order of magnitude less than the pump intensity. The anisotropic transient transmission induced by the pump pulse was measured by electro-optic 

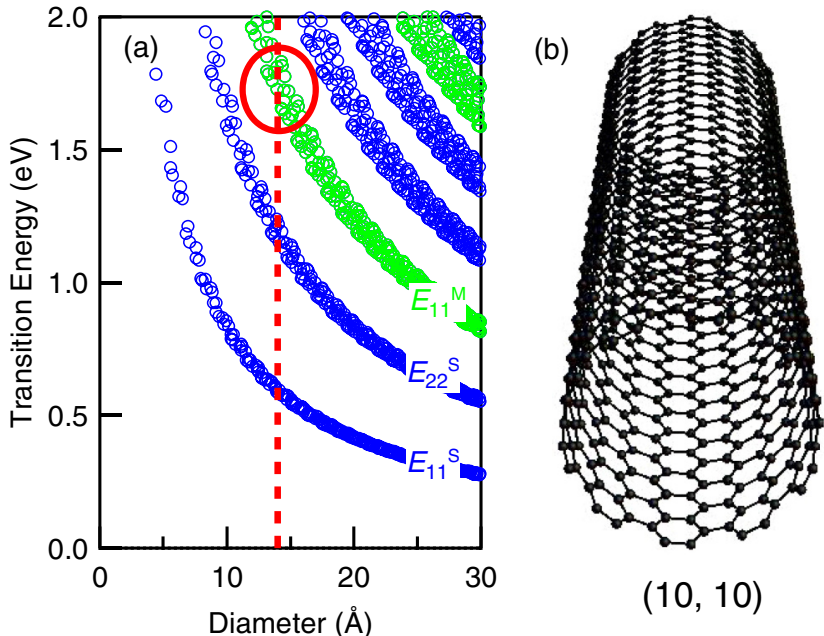

$(10,10)$
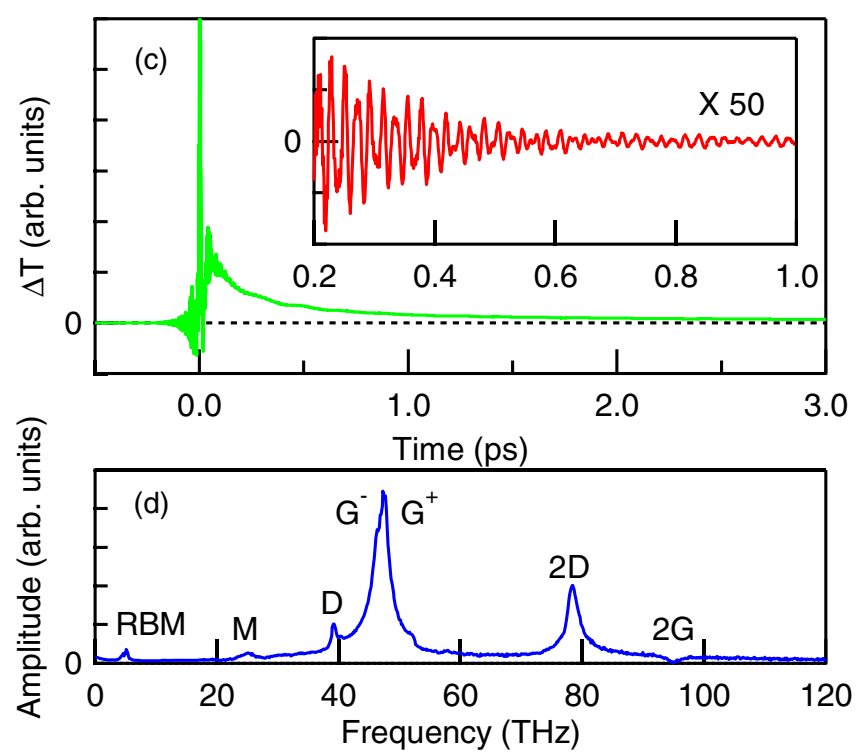

FIG. 1. (Color online) (a) Calculated optical transition energy as a function of tube diameter. Each mark corresponds to one specific chirality. The notations $E_{11}^{\mathrm{S}}, E_{22}^{\mathrm{S}}$, and $E_{11}^{\mathrm{M}}$ indicate the first- and second-lowest transitions corresponding to van Hove singularities for semiconducting (S) and metallic (M) CNTs. The average diameter of the sample is indicated by the dashed vertical line. (b) Typical structure of M-SWCNTs used in this study. (c) Transient transmittance of M-SWCNTs as a function of the pump-probe delay time. The inset shows the high-frequency components after subtracting the electronic contributions. (d) Fourier-transformed spectrum of the oscillatory components observed in (c). Typical phonon peaks are marked in the figure.

sampling. The time delay between pump and probe was controlled using an optical shaker with a 15-ps scanning range at a $20-\mathrm{Hz}$ repetition rate. The signal was amplified by a current amplifier and analyzed by a personal computer using an analog-to-digital converter. A bandpass filter with a central wavelength ranging from 700 to $980 \mathrm{~nm}$ and a bandwidth of $10 \mathrm{~nm}$ was placed behind the sample to detect wavelength-resolved signals. We also measured spontaneous Raman spectra of M-SWCNTs and highly oriented pyrolytic graphite (HOPG) using a conventional Raman spectrometer at an excitation wavelength of $785 \mathrm{~nm}(1.58 \mathrm{eV})$ (inVia Reflex,
Renishaw). All the measurements were performed at room temperature.

Figure 1(c) shows the observed transient transmission change $(\Delta T)$ of the M-SWCNTs. A strong instantaneous electronic response emerges near the time origin, and subsequently the decay of photoexcited carriers and thermal relaxation follows on a picosecond time scale. After subtracting these electronic contributions through a double-exponential fitting procedure, the high-frequency coherent oscillations can be observed as shown in the inset of Fig. 1(c). The Fouriertransformed (FT) spectrum of the oscillations, which is shown in Fig. 1(d), reveals six main peaks located at the well-known phonon frequencies of CNTs: the RBM $(5.17 \mathrm{THz})$ and $M$ $(25.2 \mathrm{THz}), D(39.2 \mathrm{THz}), G^{ \pm}(47.3 \mathrm{THz}), 2 D(78.3 \mathrm{THz})$, and $2 G(94.7 \mathrm{THz})$ modes [18]. The high-frequency second-order modes (the $2 D$ and $2 G$ modes) are observed with visible signal amplitude. Note that these modes are generated via the second-order Raman process or double-resonant Raman scattering [18,25], and therefore the observed signals do not correspond to the amplitude of the $2 D$ and $2 G$ phonons but are associated with the covariance, which can be regarded as the squeezed phonons [26-28].

Because coherent phonons are usually generated by the difference frequency of the driving field via impulsive stimulated Raman scattering, the amplitudes of the high-frequency coherent phonons become rather weak because of the limited bandwidth of incident laser pulses. For example, in HOPG, the amplitude of the $2 D$ mode $(80 \mathrm{THz})$ observed by coherent phonon spectroscopy is much smaller than that observed by conventional Raman spectroscopy, as shown in Figs. 2(a) and 2(b). In contrast, the amplitude of the $2 D$ mode in M-SWCNTs observed by coherent phonon spectroscopy is much stronger than that measured by the conventional Raman spectroscopy as shown in Figs. 2(c) and 2(d). This indicates that an unknown resonance effect contributes to the strong signal enhancement of high-frequency second-order coherent phonons in M-SWCNTs.

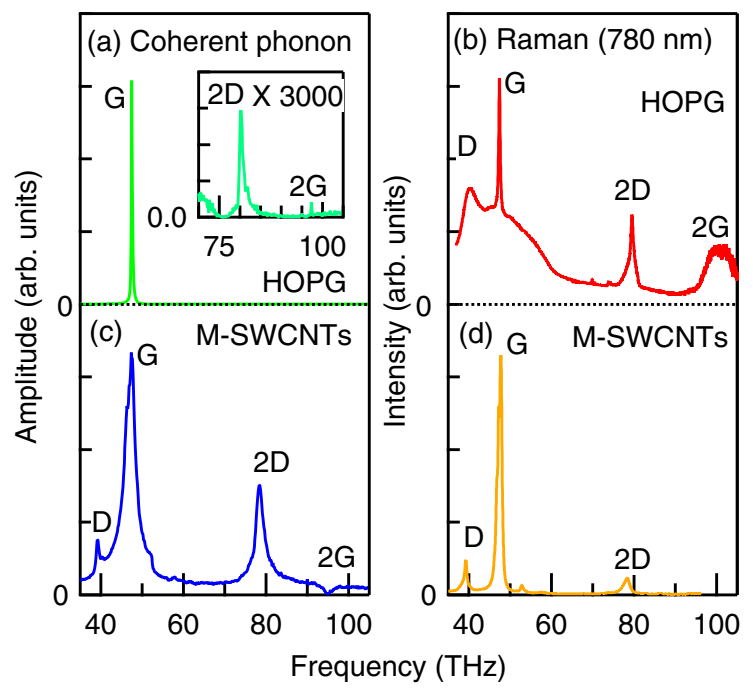

FIG. 2. (Color online) (a) Coherent phonon and (b) Raman spectra of HOPG and (c) coherent phonon and (d) Raman spectra of M-SWCNTs. The inset is a magnification of high-frequency components of HOPG, observed by coherent phonon measurement. 


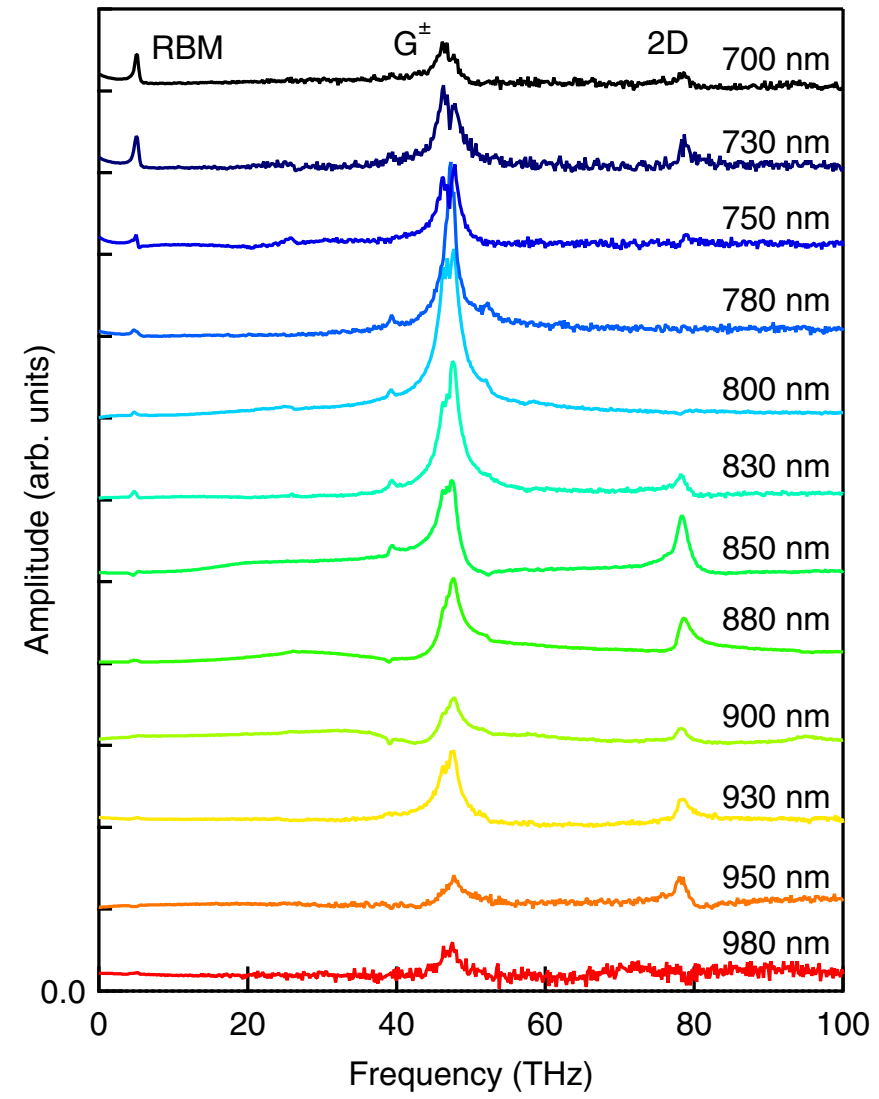

FIG. 3. (Color online) Probe-wavelength dependence of the coherent phonons in M-SWCNTs. Values indicate the center wavelengths of bandpass filters with a bandwidth of $10 \mathrm{~nm}$.

To observe the resonant effects in the probing process of coherent phonons, we resolve the spectrum of the probe pulse using bandpass filters. Figure 3 shows the FT spectra observed at probe wavelengths ranging from 700 to $980 \mathrm{~nm}$. The FT amplitudes for the RBM and $G$ and $2 D$ modes are sensitive to the probe wavelength. To highlight the signal enhancement clearly, the probe-wavelength dependence of the FT amplitudes of the RBM and $G$ and $2 D$ modes are summarized in Fig. 4(a). The amplitudes of the RBM and $G$ and $2 D$ modes are resonantly enhanced at 730, 780, and 850 $\mathrm{nm}$, respectively. Our experimental apparatus also allows us to extract phase information on the observed coherent phonons as a function of probe wavelength. We found that the phase of each mode shifts gradually with the probe wavelength, similar to the previous results observed in the resonant coherent Raman processes $[13,14]$. The phase shift most likely comes from a modulation of the dielectric constant induced by photoexcited carriers, although further investigation is needed to clarify the origin.

According to the Kataura plot [Fig. 1(a)] [17] and the absorption spectrum [22], the lowest van Hove singularity for the 1.4-nm-diameter M-SWCNTs is located at around $700 \mathrm{~nm}$ $(\sim 1.74 \mathrm{eV})$, which is higher in energy compared with the central wavelength of the laser $(800 \mathrm{~nm}, 1.55 \mathrm{eV})$. Therefore, the observed resonance enhancement of the RBM and $G$ and $2 D$ modes comes from a resonance of the stimulated Stokes process, in which the sum frequency of the scattered light and
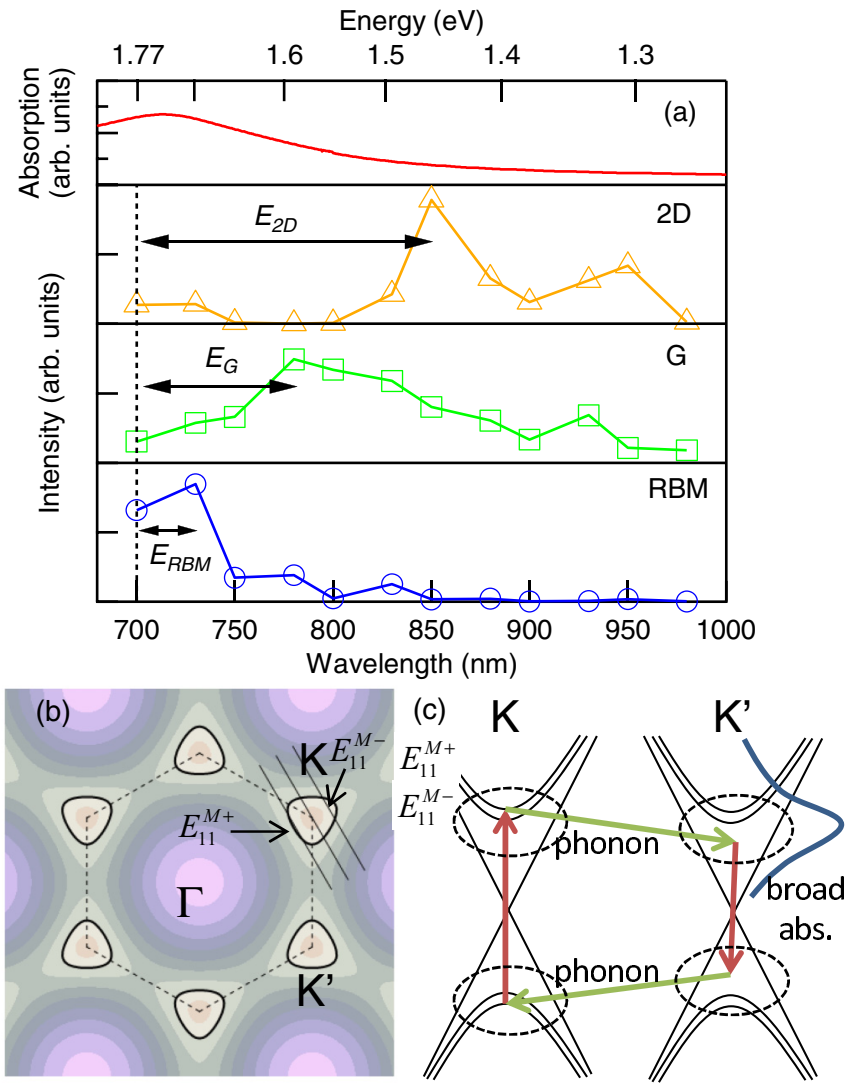

FIG. 4. (Color online) (a) Fourier-transformed amplitudes of the $2 D, G$, and RBM modes as a function of the probe wavelength together with the lowest absorption band of metallic carbon nanotubes. The $G^{+}$and $G^{-}$modes are not resolved in this analysis. (b) Schematic of the energy landscape of electronic states in graphene. The thin solid lines show the cutting lines for the energy levels of M-SWCNTs, in which the energy minima along this line correspond to the van Hove singularities. Due to the trigonal warping effect illustrated by the distortion from the circular equienergy line, the singularity splits into $E_{11}^{\mathrm{M}+}$ and $E_{11}^{\mathrm{M}-}$ in the case of M-SWCNTs that broadens the absorption spectrum. (c) Schematic of a possible resonant process that contributes to the enhancement of the $2 D$ mode. The intermediate states of the second-order Raman process may both be resonant to the broad resonant absorption of M-SWCNTs, which may play an important role for the observed enhancement of the $2 D$ mode.

each phonon is in resonance with the van Hove singularities. Given the energies of these modes $\left(\hbar \omega_{\mathrm{RBM}}=21 \mathrm{meV}, \hbar \omega_{\mathrm{G}}=\right.$ $190 \mathrm{meV}$, and $\left.\hbar \omega_{2 \mathrm{D}}=320 \mathrm{meV}\right)$, the resonant peaks should be located at around $1.72 \mathrm{eV}(\sim 721 \mathrm{~nm}), 1.54 \mathrm{eV}(\sim 808 \mathrm{~nm})$, and $1.41 \mathrm{eV}(\sim 882 \mathrm{~nm})$, respectively. These values are in quite good agreement with the observed amplitude maxima of all the listed modes, as shown by solid arrows in Fig. 4(a). These facts may well explain the resonant wavelength of these three modes. We could also see small resonant peaks observed at 950 and $930 \mathrm{~nm}$ in the $2 D$ and $G$ modes, respectively, which might be attributed to residual semiconducting CNTs.

It is notable that the RBM and $G$ and $2 D$ modes have different resonant shapes; the RBM has a narrow resonant shape, whereas the $G$ and $2 D$ modes seem to have a broader one. The similar trend is also seen in the FT spectra as shown in Fig. 3. This result is consistent with that observed previously [14], and 
may originate from the difference in electron-phonon coupling strength. The large electron-phonon coupling is expected to make a short dephasing time for the corresponding coherent phonon around its resonant wavelength. In case of the RBM, the dephasing time of $\sim 2$ ps was observed around the resonant wavelength. This relatively long dephasing time produces a small damping rate, and then results in the narrow resonant peak. On the other hand, very short dephasing times of 0.15 and 0.2 ps were observed for the $G$ and $2 D$ modes, respectively, as a result of the strong electron-phonon coupling for these modes [29]. The large electron-phonon coupling gives rise to a large damping rate, leading to the broader resonant shape of the $G$ and $2 D$ modes.

Finally, we discuss the strong enhancement of the coherent $2 D$ mode in M-SWCNTs. As shown in Figs. 2(a) and 2(c), this mode shows a much stronger enhancement than that in HOPG. Furthermore, as shown in Figs. 2(c) and 2(d), the amplitude ratio between the coherent $2 D$ and $G$ modes $\left(A_{2 \mathrm{D}} / A_{\mathrm{G}}\right)$ is much higher than that observed in conventional Raman spectroscopy at $780 \mathrm{~nm}$, suggesting that the second-order $2 D$ mode has an additional enhancement factor compared with the first-order Raman modes.

This additional enhancement could be explained by the efficient excitation process of the second-order $2 D$ mode in M-SWCNTs. First, the lowest optical transition associated with the van Hove singularities in M-SWCNTs has a small energy splitting due to the trigonal warping effect, as shown in Fig. 4(b) [18], whose value might be close to the phonon energy [30]. Resultantly, the two separated optical transitions,
$E_{11}^{\mathrm{M}+}$ and $E_{11}^{\mathrm{M}-}$, contribute to the broadening of the absorption spectrum as schematically shown in Fig. 4(c). Because the $2 D$ mode is excited via the second-order Raman scattering process [31], the number of the resonant denominators is twice as large as that in the first-order Raman process; the two intermediate states could be resonant to the broad absorption spectrum as shown by dotted circles in Fig. 4(c). This leads to the additional enhancement for the $2 D$ mode that results in the strong coherent phonon amplitude of the $2 D$ mode observed in our experiment. Second, the $2 D$ mode is related to phonons at the $K$ point in the Brillouin zone, as shown by the horizontal arrows in Fig. 4(c), where strong electron-phonon coupling is expected owing to the Kohn anomaly [19]. This strong coupling could result in a large Raman-scattering cross section for the $2 D$ mode. These two factors may lead to much higher enhancement of the observed coherent $2 D$ mode than of the first-order Raman modes in M-SWCNTs.

In summary, we have measured the high-frequency coherent phonons in M-SWCNTs. In addition to the first-order coherent phonons such as the RBM and $M$ and $G$ modes, we clearly observed the second-order $2 D$ mode thanks to a strong resonance enhancement. This effect can be qualitatively understood by taking account of both the second-order resonance of the $2 \mathrm{D}$ mode at the broad absorption peak of van Hove singularities and the strong electron-phonon coupling due to the Kohn anomaly in M-SWCNTs.

This work is partly supported by KAKENHI (Grants No. 26107517, No. 25800177, No. 25600113, and No. 23241034).
[1] J.-H. Kim, A. R. T. Nugraha, L. G. Booshehri, E. H. Hároz, K. Sato, G. D. Sanders, K.-J. Yee, Y.-S. Lim, C. J. Stanton, R. Saito, and J. Kono, Chem. Phys. 413, 55 (2013).

[2] T. Dekorsy, G. C. Cho, and H. Kurz, Light Scattering in Solids VIII 76, 169 (2000).

[3] Coherent Vibrational Dynamics (Practical Spectroscopy), edited by G. Lanzani, G. Cerullo, and S. D. Silvestri (CRC, Boca Raton, 2007).

[4] M. Hase, M. Kitajima, S.-I. Nakashima, and K. Mizoguchi, Phys. Rev. Lett. 88, 067401 (2002).

[5] K. Makino, J. Tominaga, and M. Hase, Opt. Exp. 19, 1260 (2011).

[6] K. W. Kim, A. Pashkin, H. Schäfer, M. Beyer, M. Porer, T. Wolf, C. Bernhard, J. Demsar, R. Huber, and A. Leitenstorfer, Nat. Mater. 11, 497 (2012)

[7] J.-H. Kim, K.-J. Han, N.-J. Kim, K.-J. Yee, Y.-S. Lim, G. D. Sanders, C. J. Stanton, L. G. Booshehri, E. H. Hároz, and J. Kono, Phys. Rev. Lett. 102, 037402 (2009).

[8] J. Hu, O. V. Misochko, A. Goto, and K. G. Nakamura, Phys. Rev. B 86, 235145 (2012).

[9] H. J. Zeiger, J. Vidal, T. K. Cheng, E. P. Ippen, G. Dresselhaus, and M. S. Dresselhaus, Phys. Rev. B 45, 768 (1992).

[10] Y.-X. Yan, E. B. Gamble Jr., and K. A. Nelson, J. Chem. Phys. 83, 5391 (1985).

[11] T. E. Stevens, J. Kuhl, and R. Merlin, Phys. Rev. B 65, 144304 (2002).
[12] Y.-S. Lim, K.-J. Yee, J.-H. Kim, E. H. Hároz, J. Shaver, J. Kono, S. K. Doorn, R. H. Hauge, and R. E. Smalley, Nano Lett. 6 , 2696 (2006).

[13] K. Mizoguchi, R. Morishita, and G. Oohata, Phys. Rev. Lett. 110, 077402 (2013).

[14] L. Lüer, C. Gadermaier, J. Crochet, T. Hertel, D. Brida, and G. Lanzani, Phys. Rev. Lett. 102, 127401 (2009).

[15] T. Kobayashi, Z. Nie, J. Du, K. Okamura, H. Kataura, Y. Sakakibara, and Y. Miyata, Phys. Rev. B 88, 035424 (2013).

[16] I. Katayama, K. Sato, S. Koga, J. Takeda, S. Hishita, H. Fukidome, M. Suemitsu, and M. Kitajima, Phys. Rev. B 88, 245406 (2013)

[17] H. Kataura, Y. Kumazawa, Y. Maniwa, I. Umezu, S. Suzuki, Y. Ohtsuka, and Y. Achiba, Synth. Metals 103, 2555 (1999).

[18] M. S. Dresselhaus, G. Dresselhaus, R. Saito, and A. Jorio, Phys. Rep. 409, 47 (2005).

[19] S. Piscanec, M. Lazzeri, F. Mauri, A. C. Ferrari, and J. Robertson, Phys. Rev. Lett. 93, 185503 (2004).

[20] K. Kato, K. Ishioka, M. Kitajima, J. Tang, R. Saito, and H. Petek, Nano Lett. 8, 3102 (2008).

[21] A. Gambetta, C. Manzoni, E. menna, M. Meneghetti, G. Cerullo, G. Lanzani, S. Tretiak, A. Piryatinski, A. Saxena, R. L. Martin, and A. R. Bishop, Nat. Phys. 2, 515 (2006)

[22] K. Yanagi, R. Moriya, Y. Yomogida, T. Takenobu, Y. Naitoh, T. Ishida, H. Kataura, K. Matsuda, and Y. Maniwa, Adv. Mater. 23, 2811 (2011). 
[23] K. Yanagi, Y. Miyata, and H. Kataura, Appl. Phys. Exp. 1, 034003 (2008)

[24] K. Yanagi, H. Udoguchi, S. Sagitani, Y. Oshima, T. Takenobu, H. Kataura, K. Matsuda, and Y. Maniwa, ACS Nano 4, 4027 (2010)

[25] C. Thomsen and S. Reich, Phys. Rev. Lett. 85, 5214 (2000).

[26] X. Hu and F. Nori, Phys. Rev. Lett. 79, 4605 (1997).

[27] G. A. Garrett, A. G. Rojo, A. K. Sood, J. F. Whitaker, and R. Merlin, Science 275, 1638 (1997).
[28] S. L. Johnson, P. Beaud, E. Vorobeva, C. J. Milne, É. D. Murray, S. Fahy, and G. Ingold, Phys. Rev. Lett. 102, 175503 (2009).

[29] G. G. Samsonidze, E. B. Barros, R. Saito, J. Jiang, G. Dresselhaus, and M. S. Dresselhaus, Phys. Rev. B 75, 155420 (2007).

[30] K. Sato, R. Saito, J. Jiang, G. Dresselhaus, and M. S. Dresselhaus, Phys. Rev. B 76, 195446 (2007).

[31] R. Saito, A. Jorio, A. G. Souza Filho, G. Dresselhaus, M. S. Dresselhaus, and M. A. Pimenta, Phys. Rev. Lett. 88, 027401 (2001). 